\title{
LINEAR INDEPENDENCE OF ITERATES AND ENTIRE SOLUTIONS OF FUNCTIONAL EQUATIONS
}

\author{
JENS PETER REUS CHRISTENSEN AND PAL FISCHER
}

(Communicated by Irwin Kra)

\begin{abstract}
A BSTRACT. A classical result of Pólya concerning the growth of Nevanlinna's characteristics of composite functions is used to prove linear independence of some iterates. The same result of Polya is also used to show the nonexistence of entire solutions of the Feigenbaum functional equation.
\end{abstract}

1. Introduction. There are many studies about linear independence of particular sequences of functions, but we are not aware of any such a study when the elements of the sequence are generated by iteration of entire functions. The main result of this paper is to show that except for trivial cases such a sequence is always linearly independent. Our proof is based upon a result of Pólya about the growth of Nevanlinna's characteristic [9]. As a related result, it will be shown that the Feigenbaum functional equation does not have an entire solution.

In this work we are following the presentation and notations of Hayman [6]. In the next section these notations will be reviewed and a few additional results will be discussed.

2. Notations and preliminaries. If $f: \mathbf{C} \rightarrow \mathbf{C}$ is an entire function, then the Nevanlinna characteristic $T(r, f)$ has the special form

$$
T(r, f)=\frac{1}{2 \pi} \int_{0}^{2 \pi} \log ^{+}\left|f\left(r e^{i \theta}\right)\right| d \theta
$$

where

$$
\log ^{+} x= \begin{cases}0 & \text { if } 0 \leq x \leq 1 \\ \log x & \text { if } x \geq 1\end{cases}
$$

The following result can be established easily.

LEMMA 1 . If $z_{1}$ and $z_{2}$ are complex numbers with $z_{2} \neq 0$, then

$$
\left|\log ^{+}\right| z_{1} z_{2}\left|-\log ^{+}\right| z_{1}|| \leq|\log | z_{2}|| \text {. }
$$

Lemma 1 together with (1) yield the next lemma.

LEMMA 2. Let $f$ be an entire function, let $\lambda$ be a nonzero complex number and $n$ a positive integer. Then

$$
\left|T(r, f)-T\left(r, \lambda^{n} f\right)\right| \leq n|\log | \lambda|| .
$$

For completeness, we state the result of Pólya mentioned in the introduction [9].

Received by the editors March 25, 1987 and, in revised form, July 17, 1987.

1980 Mathematics Subject Classification (1985 Revision). Primary 30D05; Secondary 39B10.

Key words and phrases. Nevanlinna's characteristic, linear independence of iterates, Feigenbaum functional equation.

Work supported in part by the NSERC of Canada under grant A-8421.

(C) 1988 American Mathematical Society $0002-9939 / 88 \$ 1.00+\$ .25$ per page 
THEOREM 1 (LEMMA 2.6 OF [6]). If $f$ and $g$ are transcendental entire functions and $\phi(z)=g(f(z))$, then

$$
T(r, g) / T(r, \phi) \rightarrow 0 \quad \text { as } r \rightarrow \infty .
$$

The proof of the previous result, as given in [6], contains implicitly the following.

THEOREM 2. Assume that $g$ is a nonconstant entire function and assume that $f$ is a polynomial of degree $\geq N+1$ and let $\phi(z)=g(f(z))$. Then

$$
T(r, \phi) \geq(N / 3) T(r, g) \text { for large enough } r .
$$

In the rest of this paper, the successive iterates of a function $f$ will be denoted in the following way: $f_{2}=f \circ f, \ldots, f_{n+1}=f \circ f_{n}, \ldots$, for $n=2,3, \ldots$, and $f_{0}$ will stand for the identity mapping.

3. On the Feigenbaum functional equation. In [7] Lanford has considered the Feigenbaum functional equation of the form

$$
f(f(\lambda x))+\lambda f(x)=0 ; \quad 0<\lambda=-f(1)<1,
$$

where the solution $f$ is unimodal (i.e. $f:[-1,1] \rightarrow[-1,1], f(0)=1, f$ is strictly increasing on $[-1,0]$ and strictly decreasing on $[0,1])$, even and fairly smooth on $[-1,1]$ and such that

$$
f(0)=1, \quad f(1) \leq 0, \quad f(\lambda) \geq \lambda, \quad f^{\prime \prime}(0)<0 .
$$

It was shown in [3], that if $f$ is an even continuous solution of (6) for a fixed $\lambda$, then it follows that $f(\lambda)>\lambda$. Further properties of solutions of (6) are discussed in [1] and in [3]. A simple way to generate all continuous and unimodal solutions is presented in [4].

In [8] Lanford exhibits a solution of (6) which is analytic on a neighbourhood of the whole real axis, and he remarks that it is essentially certain that this particular solution is not entire.

To show that (6) does not have an entire solution, we shall prove the following theorem.

THEOREM 3. Let $F$ be a nonidentically zero entire function which satisfies the functional equation

$$
F(F(\lambda z))+\lambda F(z)=0
$$

for all $z \in \mathbf{C}$, where $\lambda$ is a fixed nonzero complex number, then either $F(z)=-z$ and $\lambda$ is arbitrary or $F(z) \equiv C$ with $\lambda=-1$.

PROOF. We have to distinguish three cases. It is easy to verify the statement of this theorem if $F$ is a polynomial with degree $\leq 1$. Secondly, it is immediate that (8) cannot have a polynomial solution of degree $\geq 2$.

It remains to be shown that (8) cannot have a transcendental entire solution. To do that we write (8) in the form

$$
F(z)=-(1 / \lambda) F(F(\lambda z))
$$

To follow Hayman's notation [6], we put $g(z)=-(1 / \lambda) F(z), f(z)=F(\lambda z)$ and $\phi(z)=g(f(z))=F(z)$. Let $N$ be a sufficiently large fixed positive number. Then by Theorem 1 we can conclude that

$$
T(r, \phi)=T(r, F) \geq \frac{N+1}{3} T(r, g)=\frac{N+1}{3} T\left(r, \frac{-1}{\lambda} F\right) .
$$


Hence, using the fact that $T(r, F)$ and $T(r,-(1 / \lambda) F)$ both tend to infinity as $r \rightarrow$ $\infty$, we can see that

$$
|T(r, F)-T(r,-(1 / \lambda) F)| \rightarrow \infty \quad \text { as } r \rightarrow \infty,
$$

which contradicts Lemma 2.

REMARK 1. We should remark that the impossibility of the third case in the above proof can be shown also by using a result of Ganapathy [5] about the entire solutions of some functional equations.

Now, we can show that the Feigenbaum functional equation does not have an entire solution.

THEOREM 4. The Feigenbaum equation (6) does not have an entire solution $f$ such that $f(0)=1$.

Proof. Assume that $f$ is an entire solution of (6). Then, it follows that the entire function

$$
g(z)=f(f(\lambda z))+\lambda f(z)
$$

is zero on $[-1,1]$. Therefore, $g(z)=0$ for all $z \in \mathbf{C}$, i.e. $f$ satisfies (8) for all $z \in \mathbf{C}$. But this last fact contradicts Theorem 3 .

REMARK 2. In the previous result, we did not assume that $f$ is an unimodal solution of $(6)$, only the conditions $f(0)=1$ and $0<\lambda<1$ were used to eliminate polynomial solutions of degree $\leq 1$ of $(8)$.

4. Problems from iteration theory. In this section we discuss linear independence of iterates. First, we prove the following theorem.

THEOREM 5. Let $\mathcal{I}$ be the set of all entire functions. Let $f$ be an entire function which is not a polynomial of degree $\leq 1$. Let $R_{f}: \mathscr{I} \rightarrow \mathscr{I}$ be defined by $R_{f}(g)=g \circ f$ for some $g \in \mathscr{I}$. Then $R_{f}$ has no eigenvalue $\neq\{0,1\}$ and any eigenfunction with eigenvalue 1 is a constant.

ProOF. We shall separate cases.

Case 1. It will be assumed that $f$ is a polynomial with degree $\geq 2, g$ is a nonconstant entire function, and $\lambda \in \mathbf{C}, \lambda \neq 0$, and

$$
g \circ f=\lambda g \text {. }
$$

Obviously, by iteration, we have also that

$$
g \circ f_{k}=\lambda^{k} g \text { for all } k \in N .
$$

By Theorem 2, we can conclude that

$$
T\left(r, \lambda^{k} g\right) \geq \frac{r^{k}-1}{3} T(r, g) \quad \text { for large enough } r .
$$

Also, by (3), we have that

$$
\left|T(r, g)-T\left(r, \lambda^{k} g\right)\right| \leq k \mid \log (|\lambda|) .
$$

But $T(r, g) \rightarrow \infty$ as $r \rightarrow \infty$, because $g$ is a nonconstant entire function. Hence, we obtain a contradiction when $k$ and $r$ are sufficiently large.

Case 2. It will be assumed that $f$ is a transcendental entire function. It is easy to see that if $g$ is polynomial, then $g$ cannot be a solution of (11). Now, Theorem 1 implies that the equation $g \circ f=\lambda g$ cannot have a transcendental entire solution.

We can generalize the previous result as follows. 
THEOREM 6. Let $g$ be a nonconstant entire function and let $f$ be an entire function which is not a polynomial of degree $\leq 1$. Then $\left\{g \circ f_{n}: n \geq 0\right\}$ is a linearly independent sequence of entire functions.

PROOF. Let $P(z)=\sum \lambda_{p} z^{P}$ be a polynomial with complex coefficients. Let $P(T)$ be the linear operator from the space of entire functions into itself defined by

$$
P(T)(g)=\sum \lambda_{p} g \circ f_{p}
$$

Evidently, the mapping $P \rightarrow P(T)$ is linear and multiplicative. Suppose, now, that the functions $g$ and $f$ yield a counterexample to the statement of this theorem, i.e. we have

$$
P(T)(g)=\sum \lambda_{p}\left(g \circ f_{p}\right)=0
$$

for some nonconstant polynomial $P$. Without loss of generality, we can assume that $\lambda_{0} \neq 0$. Hence, $P(z)=\prod_{i=1}^{d}\left(z-\lambda_{i}\right)$, where degree of $P=d$ and $\lambda_{i} \neq 0$ for $1 \leq i \leq d$. Using the fact that the mapping $P \rightarrow P(T)$ is multiplicative, clearly, it is enough to establish that the assumption

$$
g \circ f=\lambda g+c, \quad \lambda \neq 0, c \text { a constant }
$$

leads to a contradiction.

From (13), by iteration, we obtain that

$$
g \circ f_{k}= \begin{cases}\lambda^{k} g+\frac{\lambda^{k}-1}{\lambda-1} c & \text { for } \lambda \neq 1, \\ \lambda^{k} g+k c & \text { for } \lambda=1\end{cases}
$$

Now, (13) shows that $g$ cannot be a polynomial. Hence, without loss of generality, from now on, we can assume that $g$ is a transcendental entire function. The impossibility of the case when $f$ is a transcendental entire function can be shown directly from (12) using Theorem 1 together with the inequality $T\left(r, \sum_{v=1}^{p} f_{v}\right) \leq$ $\sum_{v=1}^{p} T\left(r, f_{v}\right)+\log p$. (This last inequality is shown in [6, p. 5], under the assumptions that the functions $f_{1}, \ldots, f_{p}$ are meromorphic.)

Hence, the only case is left to be considered, when $f$ is a polynomial of degree $p$, with $p \geq 2$. By Theorem 2 , we can see that

$$
T\left(r, g \circ f_{k}\right) \geq \frac{p^{k}-1}{3} T(r, g) .
$$

Let

$$
h= \begin{cases}\lambda^{k} g+\frac{\lambda^{k}-1}{\lambda-1} c & \text { for } \lambda \neq 1, \\ \lambda^{k} g+k c & \text { for } \lambda=1 .\end{cases}
$$

Obviously $T(r, h)$ grows no faster (in terms of $k$ ) then $T(r, g)$ plus a polynomial of degree 1 in $k$. Therefore, (14) leads to a contradiction if $k$ and $r$ are sufficiently large. This completes the proof of this theorem.

\section{REFERENCES}

1. M. Cosnard, Étude des solutions de l'équation de Feigenbaum, Astérisque 98-99 (1982), 143162.

2. P. Fischer, Feigenbaum functional equation and periodic points, Aequationes Math. 30 (1986), 202-207. 
3. __ Feigenbaum functional equations as dynamical systems, Chaos, Fractals, and Dynamics (P. Fischer and W. R. Smith, eds.), Dekker, 1985, pp. 183-188.

4. $\ldots$, On the general unimodal solution of the Feigenbaum functional equation and related classes of solutions (to appear).

5. V. Ganapathy Iyer, On a functional equation. II, Indian J. Math. 2 (1960), 1-7.

6. W. K. Hayman, Meromorphic functions, Oxford Mathematical Monograph, Oxford at the Clarendon Press, 1964.

7. O. E. Lanford III, Smooth transformations of intervals, Séminaire Bourbaki, 1980/81, Lecture Notes in Math., vol. 901, Springer-Verlag, Berlin and New York, 1981, pp. 36-54.

8. _ A computer-assisted proof of the Feigenbaum conjectures, Bull. Amer. Math. Soc. (N.S.) 6 (1982), 427-434.

9. G. Pólya, On an integral function of an integral function, J. London Math. Soc. 1 (1926), 12-15.

MATEMATISK INSTITUT, KøBEnhaVNS UNIVERSitet, UNIVERSitetsPaRKEN 5, DK2100 KøBENHAVN $\varnothing$, DENMARK

DEPARTMENT OF MATHEMATICS AND STATISTICS, UNIVERSity OF GUELPH, GUELPH, Ontario, Canada N1G 2W1 (Current address of Pal Fischer)

Current address (J. P. R. Christensen): KTAS, UN, NøRREGADE 21, 1199 KøBENHAVN K, DENMARK 